\title{
Genetic tools for increased white clover seed production
}

\author{
B.A. BARRETT ${ }^{1}$, I.J. BAIRD ${ }^{2}$ and D.R. WOODFIELD ${ }^{1}$ \\ ${ }^{1}$ AgResearch, Grasslands Research Centre, PB 11008, Palmerston North \\ ${ }^{2}$ AgResearch, Canterbury Agriculture and Science Centre, P.O. Box 60, Lincoln
}

brent.barrett@agresearch.co.nz

\begin{abstract}
Genome maps and DNA markers allow plant breeders to decipher the genetic control of commercially vital quantitative traits. Discovery of the components of these genetic control networks will create opportunities to implement more efficient forage breeding systems. Gene mapping research strategies, using a combination of field trials and DNA-marker data, were used to discover quantitative trait loci (QTL) impacting seed production in white clover (Trifolium repens). Twelve QTL influencing seed yield (SY) or the two component factors inflorescence density (ID) and seed provisioning (SP) were detected using a full-sib gene mapping population in the 2002 trial at Lincoln, New Zealand. Three of these QTL also were detected in the 2003 trial, of which one for ID and one for SP harboured alleles that accounted for up to a $50 \%$ change in ID and SP trait value, respectively. Genetic tests for these two alleles were used in a marker-assisted selection (MAS) experiment to identify the plants in the gene mapping population with the highest commercial potential. This initial proof-ofconcept MAS design was successful in identifying the subpopulation with the highest SY value. Implementation of MAS designs will result in increased ability for plant improvement programmes to assure development of commercially successful forage cultivars.
\end{abstract}

Keywords: marker-assisted selection, QTL, seed production, white clover

\section{Introduction}

The commercial success of white clover cultivars is increasingly dependent on strong seed production potential. However, field screening to select for seed production is expensive, time consuming, and often blurred by environment-specific effects. Utilisation of molecular marker technologies will substantially enhance industry's ability to market forage cultivars with improved performance for cornerstone traits, without the use of genetic engineering.

Discovery and characterisation of the genetic factors controlling seed yield in white clover, and estimation of their stability across environments should shorten time and reduce costs required to commercialise new cultivars. These factors may be discovered using the combination of field-based and laboratory-based genetic experiments. Recently field-based experiments have demonstrated the independent genetic control of inflorescence density (ID, inflorescences/plant) and seed provisioning (SP, grams seed/inflorescence), two key characters underpinning the commercially important trait, seed yield (SY) in white clover (Woodfield et al. 2004). SP is a complex character influenced by floret number, ovule fertility, and thousand seed weight.

Widdup et al. (2004) reports response to selection for ID and SP in the development of clover varieties with superior seed yield potential. An important conclusion drawn from their work is that within agronomically superior populations, SY potential can be enhanced to a commercially viable level without compromising agronomic performance (Widdup et al. 2004).

Expression of quantitative plant traits, such as ID and SP, is under polygenic control. The more that is understood about the genes which control these traits, the greater the opportunity to implement breeding strategies to maximise genetic gain. Gene mapping employs a combination of field and laboratory science to test experimental populations and define the location and effect of genes. Each polygene location is referred to as a quantitative trait locus (QTL), and accounts for a proportion of the variation for that trait. Within an individual plant, there are two variants for each QTL. Each variant is referred to as an allele, and it is the allele combination which determine whether the QTL's influence on performance is positive, negative, or neutral. QTL often act to influence other QTL, resulting in multifaceted networks of genetic control. In addition to this complex network of genes, trait expression is profoundly influenced by environmental signals such as plant nutrient availability, and climatic variability. The sum of these genetic and environment components, and all possible interactions among them dictate the expression of a quantitative trait, such as SY, in individual plants.

Microsatellites, a preferred DNA marker technology for gene mapping, have been developed for white clover using proprietary DNA sequence databases (Faville et al. 2003; Sawbridge et al. 2003). These markers have been used to build a comprehensive map describing 500 locations in the white clover genome (Barrett et al. 2004), that can be scanned at high resolution for QTL.

QTL discovery underpins marker-assisted selection (MAS), a breeding strategy that combines DNA testing with field trials to maximise gain from selection. These 
DNA tests use marker data at QTL to identify those individuals with a high probability of containing the optimal genetic configuration for superior performance in target markets (Faville et al. 2003).

The objective of this research was to use field and laboratory-based gene mapping techniques to discover QTL which regulate ID and SP, two genetic determinants of seed yield in white clover. It also demonstrates a MAS strategy using genetic tests to identify white clover plants with high commercial potential.

\section{Methods}

\section{Plant material}

A population of 184 full-sib $F_{1}$ progeny was created between two phenotypically divergent, highly heterozygous white clover genotypes: 'P6525', a parent of the cultivar 'Grasslands Sustain' (Caradus et al. 1997), and 'P3647', a plant selected for nematode resistance (Mercer et al. 1999). P6525 exhibits medium to large leaves, leaf mark, many long thin stolons, and dense growth; whereas P3647 exhibits large leaves, no leaf mark, few short thick stolons, and open growth.

\section{Field trials}

The two parent plants and 184 full-sibs were evaluated at Lincoln, Canterbury for seed yield and seed yield components over two years representing two environments; the 2001/2002 growing season, and the $2002 / 2003$ growing season. Each genotype was replicated three times in a randomised complete block design. Entries were re-randomised and re-cloned for each environment. Each plot contained a single clonal copy of a genotype, and was contained by a $50 \mathrm{~cm}$ diameter ring to establish a uniform area for monitoring trait expression. Weekly observations were made of inflorescence number during the growing season, the sum of these counts was used as the number of inflorescences per plant. At maturity, each plot was harvested and seed yields and seed yield components were recorded.

\section{DNA marker data}

Data collection and analysis for all microsatellite markers were as described by Faville et al. (2003). The white clover genome map used is that reported by Barrett et al. (2004). In brief, a subset of individuals $(\mathrm{n}=92)$ in population $\mathrm{P} 3647 \times \mathrm{P} 6525$ were genotyped at 493 selected microsatellite loci to provide $98 \%$ coverage and a mean scan resolution of $1.6 \mathrm{cM} /$ marker locus throughout the white clover genome.

\section{Statistical analysis}

Seed yield (SY, g/plant), inflorescence density (ID, inflorescences/plant), and seed provisioning (SP, g/
Figure 1 The structure used for a full-sib $F_{1}$ white clover QTL discovery population, indicating parent and progeny allele configurations at a locus. Progeny allele configurations correspond to the subpopulations used to test for specific allele effects at each QTL.

\section{Parent allele configuration}

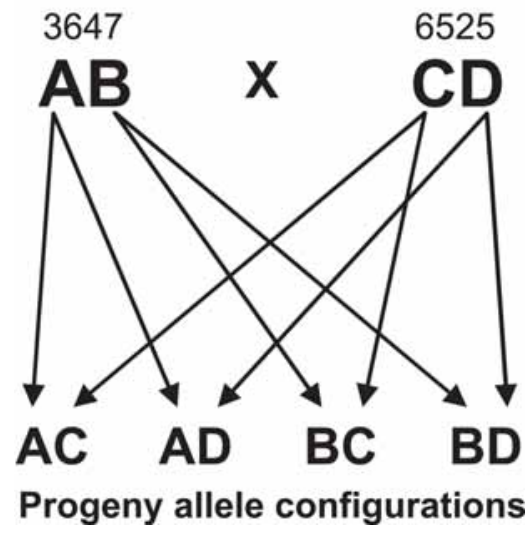

inflorescence) trait data were analysed by analysis of variance (ANOVA) using GenStat version 7. QTL discovery for each trait was done within the genotyped subset of 92 individuals on a single trial basis, using six trait datasets, SY02, SY03, ID02, ID03, SP02, and $\mathrm{SP} 03$. For each trait, the within-year mean trait value for each of the subset of 92 genotypes was used. Interval analyses were implemented in MapQTL 4.0 software (www.kyazma.nl) for QTL discovery in the subset of genotyped individuals. Statistical significance of each QTL was assessed by calculating the Log of Odds (LOD) ratio, where scores greater than 3.0 are significant; and by determining the percentage of variation in trait performance accounted for by that QTL. Parent and progeny allele configuration are described in Figure 1. Subpopulation means calculated for each marker locus were used to assess the impact of specific allele combinations on trait performance in the progeny, and identify which parent conferred the superior allele at each QTL.

\section{MAS Experiment}

Select QTL identified in the gene mapping population were used to conduct a MAS experiment to select for $\mathrm{SY}$ in the same population. This type of test is a necessary proof-of-concept prior to implementing MAS in unrelated commercial white clover populations. In this MAS experiment, genetic tests using a microsatellite marker associated with the most influential ID QTL and a microsatellite marker associated with the most influential SP QTL were used to select for plants with superior SY performance. The 
Table 1 Summary statistics of white clover seed production characters in a gene mapping population tested for seed yield, inflorescence density, and seed provisioning in two environments. ' 3647 ' and ' 6525 ' were the parents of the full-sib population used in this experiment.

\begin{tabular}{lcccccc}
\hline & \multicolumn{2}{c}{$\begin{array}{c}\text { Seed yield } \\
\text { (g/plant) }\end{array}$} & & \multicolumn{2}{c}{$\begin{array}{c}\text { Inflorescence density } \\
\text { (number/plant) }\end{array}$} & \multicolumn{2}{c}{$\begin{array}{c}\text { Seed provisioning } \\
\text { (g/inflorescence) }\end{array}$} \\
& 2002 & 2003 & 2002 & 2003 & 2002 & 2003 \\
\hline Parent 3647 & 3.9 & 9.3 & 35 & 69 & 0.098 & 0.134 \\
Parent 6525 & 6.9 & 12.5 & 40 & 69 & 0.190 & 0.181 \\
Population Mean & 18.3 & 13.5 & 121 & 107 & 0.155 & 0.132 \\
Minimum & 0.7 & 0.5 & 4 & 2 & 0.027 & 0.022 \\
Maximum & 50.2 & 34.5 & 266 & 229 & 0.304 & 0.276 \\
SEM & 0.65 & 0.46 & 3.7 & 3 & 0.004 & 0.0004 \\
\hline
\end{tabular}

Table 2 Descriptive characters of 18 QTL which impact seed production in white clover. 'Linkage Group' identifies which chromosome in the genome harbours the QTL, ‘\% Var Expl.' indicates the variation accounted when all the observed variation is tested on a per QTL basis. 'LOD' indicates the statistical validity of the QTL, scores > 3.0 are significant.

\begin{tabular}{|c|c|c|c|c|}
\hline Trait & Year & $\begin{array}{l}\text { Linkage } \\
\text { group }\end{array}$ & $\begin{array}{l}\text { \%variation } \\
\text { explained }\end{array}$ & LOD \\
\hline \multicolumn{5}{|c|}{ Seed yield } \\
\hline & 2002 & $\mathrm{~F} 2$ & 29 & 3.7 \\
\hline & 2002 & $\mathrm{C} 2$ & 27 & 5.0 \\
\hline & 2002 & D1 & 20 & 3.7 \\
\hline & 2002 & $\mathrm{~A} 2$ & 18 & 3.6 \\
\hline & 2003 & D2 & 32 & 7.6 \\
\hline \multicolumn{5}{|c|}{ Inflorescence density } \\
\hline & 2002 & $\mathrm{C} 2$ & 35 & 6.7 \\
\hline & 2002 & D2 & 32 & 3.1 \\
\hline & 2002 & D1 & 19 & 3.8 \\
\hline & 2002 & $\mathrm{E} 1$ & 17 & 3.5 \\
\hline & 2002 & C1 & 16 & 3.6 \\
\hline & 2003 & $\mathrm{C} 2$ & 26 & 4.5 \\
\hline & 2003 & E1 & 16 & 3.6 \\
\hline \multicolumn{5}{|c|}{ Seed provisioning } \\
\hline & 2002 & D2 & 22 & 5.1 \\
\hline & 2002 & F2 & 17 & 3.6 \\
\hline & 2002 & A1 & 14 & 3.0 \\
\hline & 2003 & D2 & 43 & 11.4 \\
\hline & 2003 & G1 & 24 & 5.4 \\
\hline & 2003 & $\mathrm{~A} 2$ & 18 & 3.9 \\
\hline
\end{tabular}

only criteria used for plant selection were two genetic tests, no field data and no information from other QTL were used. For each microsatellite, the testing for positive and negative alleles inherited from the parent with the greatest influence on trait value in the QTL discovery experiment were used to place the individuals in the gene mapping population into one of four subpopulations: ID-SP-, ID-SP+, ID+SP-, and $\mathrm{ID}+\mathrm{SP}+$. For each of the subpopulations, SY02 and SY03 values for each plant were used to measure individual and subpopulation mean SY performance in each trial. Subpopulation SY means were compared using an ANOVA.

\section{Results \\ Field trials}

Population performance in the 2002 trial was higher than in the 2003 trial for each trait measured in the population. In both the 2002 and 2003 trial, parent P3647 exhibited lower SY and SP than P6525, while both parents exhibited similar values for ID (Table 1). Substantial variation for each of the three traits was observed among individuals. SY02 and SY03 values exhibited a wide range, from 0.7 to $50.2 \mathrm{~g} /$ plant and 0.5 to $34.5 \mathrm{~g} / \mathrm{plant}$, respectively. ID02 and ID03 ranged from 4 to 266 and 2 to 229 inflorescences/plant, respectively. The range of values observed for SP02 and SP03 was less extreme, from 0.027 to 0.304 and 0.022 to $0.276 \mathrm{~g}$ /inflorescence, respectively (Table 1). The ANOVA indicated significant genotype, environment, replicate, and genotype $\mathrm{x}$ environment effects for SY, ID, and SP.

\section{QTL discovery}

A gene mapping analysis of the white clover genome resulted in identification of 18 QTL distributed across 9 linkage groups (chromosomes), and revealed unique QTL controlling each trait (Table 2). There were wide differences in the proportion of trait variation accounted for by QTL, with values ranging from $14 \%$ to $43 \%$. LOD scores ranged from 3.0 to 11.4 , and were correlated with the proportion of trait variation accounted for $\left(r^{2}=0.65\right)$.

An example of a QTL discovered in this study is depicted in Figure 2, where the LOD score, which measures the genetic impact on trait variation is plotted versus location within a specific linkage group. This QTL is located on linkage group A2 in the white clover genome and most likely is near marker prs 497b, whose genome location most closely corresponds with the peak in the LOD score profile.

Data from the 2002 trial revealed 12 QTL; four, five, and three for SY02, ID02, and SP02, respectively (Table 
Figure 2 An example of a seed production QTL discovered in the white clover genome using population P3647 $x$ P6525. The top portion of the figure plots LOD score vs. genome location for trait SY02 within linkage group A2, and identifies a specific QTL peak in the central portion. The group A2 map is shown below the chart.

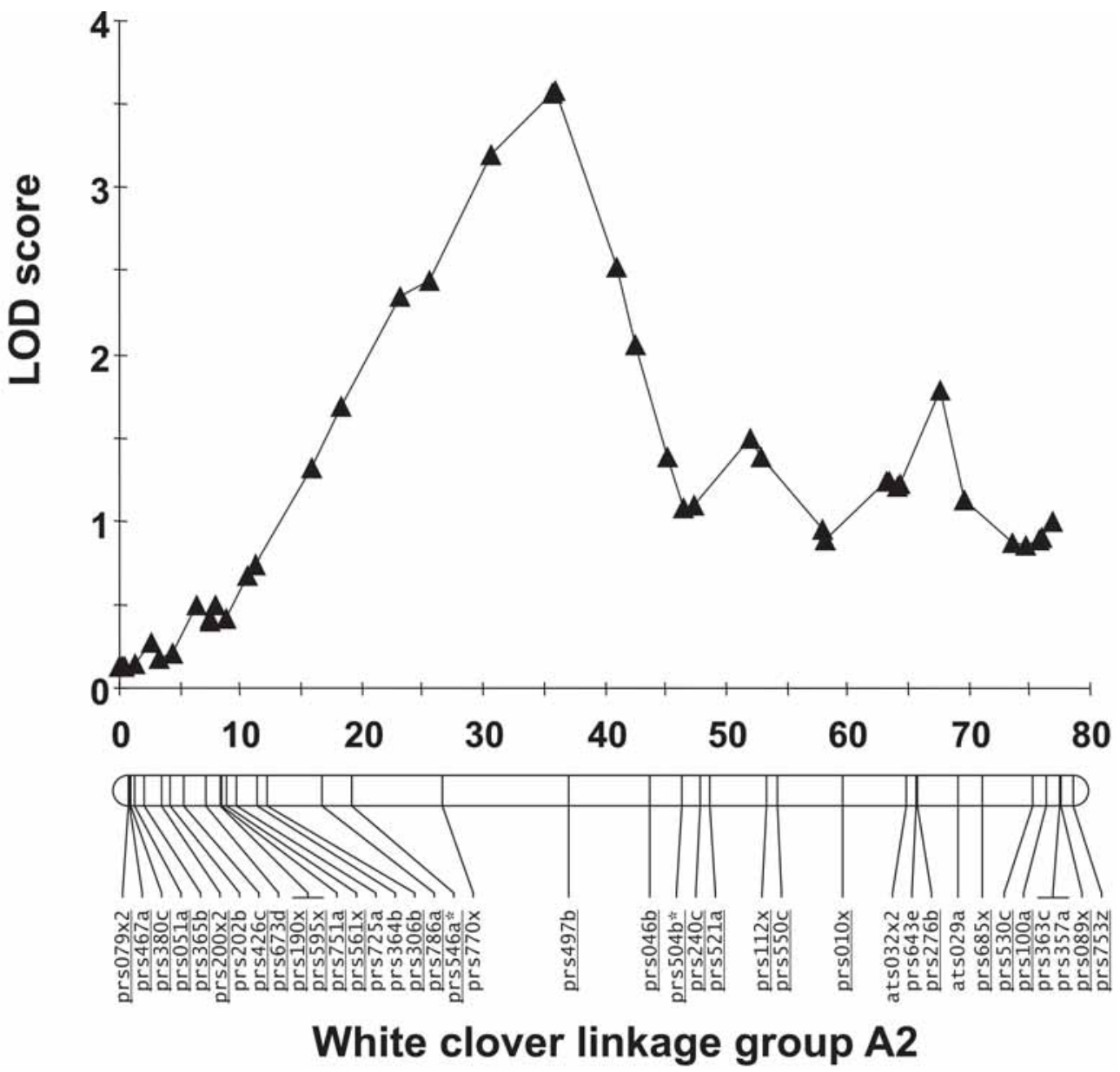

2). Two SY02 QTL on linkage groups C2 and D1 were in a similar location to QTL influencing ID02 on the same linkage groups, and one SY02 QTL on linkage group F2 was in a similar location to a QTL controlling SP02 on F2. Examination of the 2003 data revealed fewer QTL, with only six detected, comprised of one, two, and three for SY03, ID03, and SP03, respectively. The sole SY03 QTL was aligned with a SP03 QTL on linkage group D2.

None of the SP QTL were in the same genome location as ID QTL in either year. Although QTL for SP02 and ID02 were discovered on linkage group D2, the QTL peaks are separated by $\sim 30$ centimorgans, suggesting that they are separate QTL. The most variation accounted for by a QTL was for the SP03 trait, with a QTL on linkage group D2 accounting for $43 \%$ of the variation. None of the SY02 QTL were detected in the 2003 trial.
However, two of the five ID02 QTL were detected again in the 2003 trial, and one of the SP02 QTL was detected in the 2003 trial.

\section{Identification of QTL for use in MAS}

Among 12 QTL detected in the 2002 trial, three were detected in the 2003 trial. A QTL for ID located on linkage group $\mathrm{C} 2$ was detected in both years, and accounted for $35 \%$ and $26 \%$ of the observed variation in each year, respectively. A second QTL for ID, located on group E1 also was detected in both environments. However, this QTL accounts for substantially less variation, $17 \%$ and $16 \%$ respectively in the two years, and was excluded from further consideration on this basis. A third QTL detected in both environments influenced SP, and is located on linkage group D2. This QTL accounted for $22 \%$ and $43 \%$ of the observed 
Figure 3 The impact of allele combinations at two QTL related to seed production in two trials (2002 and 2003). The effects were similar in magnitude in both trials, indicating these alleles may be useful for the design of genetic tests for marker-assisted selection in commercial seed production trails. a) the four allele combinations at the ID QTL on linkage group C2, demonstrating that allele D is consistently superior in performance to allele C. b) the four allele combinations at the SP QTL on linkage group D2, demonstrating that allele $\mathrm{B}$ is consistently superior in performance to allele $\mathrm{A}$.

a)

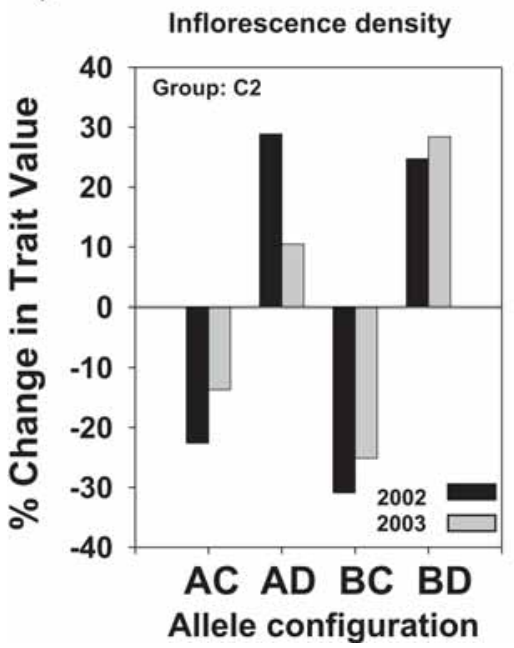

b)

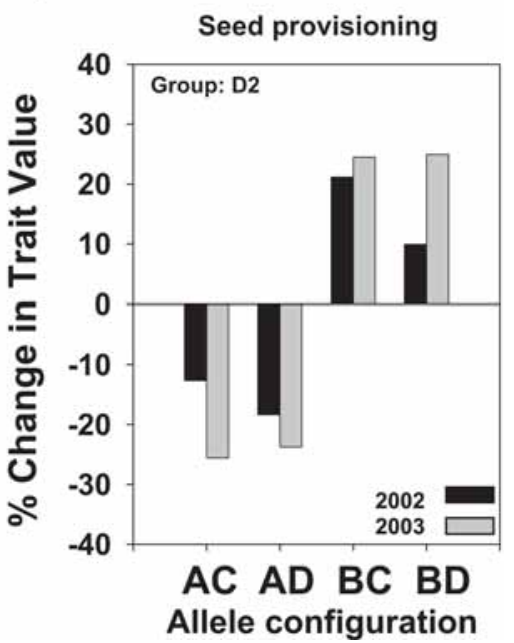

Figure 4 Results of a marker-assisted selection (MAS) experiment in white clover, demonstrating successful selection of plants with superior performance using DNA tests. Selection criteria were two genetic tests, one for an ID allele and one for an SP allele. Bars with different letters indicate values are significantly different within year.

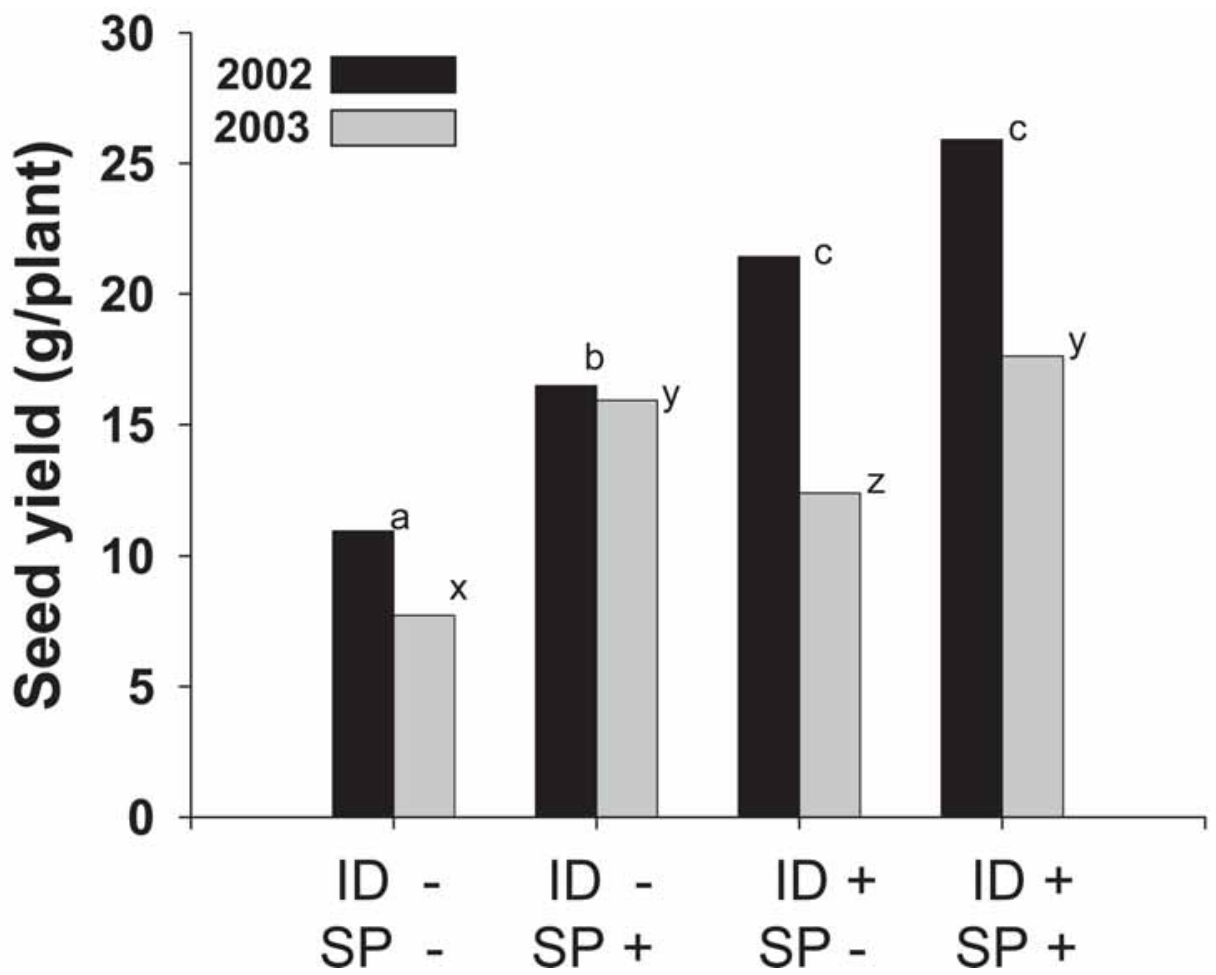


variation in SP02 and SP03, respectively.

For each QTL identified in both environments, subpopulation means reveal clear patterns of correlation between allele configuration at the QTL and trait performance. For the C2 QTL, the subpopulation with allele combination $\mathrm{BD}$ exhibited the highest ID values, while the subpopulation with allele configuration $\mathrm{BC}$ exhibited the lowest values (Figure 3a). This indicates that parent 6525 is heterozygous at a QTL controlling ID, and allele D is substantially superior to allele $\mathrm{C}$ at that QTL. In each environment, allele D conferred a $\sim 50 \%$ increase in ID relative to allele C. This QTL may also be polymorphic in $\mathrm{P} 3647$, however the positive trait value difference attributed to the $B$ allele is only noted in 2003 data and contributes less to performance than the D allele.

The SP QTL detected on D2 accounts for a substantial change in trait values, with the $\mathrm{B}$ allele conferring a $\sim 50 \%$ increase in SP03 compared to the A allele (Figure $3 b)$. This indicates that the $\mathrm{P} 3647$ is heterozygous at this QTL. The $\mathrm{C}$ and $\mathrm{D}$ alleles appear to have little influence on performance, however the most consistent performance was by the subpopulation with allele combination BC.

\section{MAS experiment}

We used the two most influential QTL discovered in the gene mapping population to implement the initial proofof-concept MAS trial for the economic trait of interest (SY) in the mapping population. Selection criteria were allele composition at the C2 QTL for ID and the D2 QTL for SP detected in both years. Selection was solely on the basis of genotype at the two QTL loci, i.e. the superior (ID+, allele D) versus inferior (ID-, allele C) variant for the ID QTL on $\mathrm{C} 2$, and the superior ( $\mathrm{SP}+$, allele B) versus inferior (SP-, allele A) variant for the SP QTL on D2. Allele configurations at the other 14 QTL were not accounted for.

In both years, the subpopulation with both superior alleles (ID+SP+) exhibited the highest mean SY, and the subpopulation with both inferior alleles (ID-SP-) exhibited the lowest mean SY (Figure 4). Selection for the superior allele at one QTL and the inferior allele at the other QTL resulted in intermediate SY values. The ANOVA detected significant $(\mathrm{P}<0.001)$ subpopulation effects for SY in each environment; however, subpopulations ID+SP+ and ID+SP- in the 2002 environment were not statistically different, and subpopulations ID+SP+ and ID-SP+ in the 2003 environment were not statistically different (Figure 4).

\section{Discussion}

This is the first report of gene mapping strategies to discover white clover QTL that consistently influence
ID and SP, the component traits of seed yield. Two of the discovered QTL were subsequently used in MAS experiments, in which a simple DNA test at two QTL was used to identify plants with high seed production value.

\section{Field trials}

The higher seed production in the 2002 trial compared with 2003 reflected the typical reduction in vigour as plants age. Surprisingly, the parent plants had higher seed production values in the 2003 environment although they were significantly lower than population mean for SY and ID in both environments (Table 1). This anomaly may be attributed in part to the age and virus load of the parent plants (continually cloned for 3-7 years) relative to the age of the full-sib progeny (1 year).

Despite the parent genotypes expressing similar values for traits such as ID, the full-sib progeny population exhibited surprisingly wide (up to 70 -fold) variation for both SY and ID. These results suggest that in outbreeding species such as white clover and perennial ryegrass, parent plants with similar values may be heterozygous at key QTL loci and give rise to populations useful for detecting the genetic control of commercially important traits.

\section{QTL discovery}

Unique QTL were discovered for SY, ID, and SP in both years. Among the 18 QTL discovered, transient ID and SP QTL effects may be co-located on linkage group D2. However, neither of the ID and SP QTL detected in both year's data are co-located in the genome. This result complements and extends results by Woodfield et al. (2004), which indicated that ID and SP are under independent genetic control. However, because genes (QTL) interact throughout the genome, there may still be interaction among QTL controlling these two SY components. Many SY QTL were co-located with either ID or SP QTL (e.g. linkage group D2), reiterating the known positive correlation between SY and these characters.

P3647, which demonstrated inferior SY and SP performance relative to $\mathrm{P} 6525$, was found to harbour superior alleles at some SY and SP QTL. Detection of superior alleles in inferior plants is common (Xiao et al. 1998) and demonstrates the power of QTL experiments relative to strictly field-based analyses for accumulating the most effective allele configurations. Gene mapping should also prove useful for mining novel and superior alleles from plants in forage germplasm banks, as some superior alleles may not be detected using only phenotypic analyses (Tanksley \& McCouch 1997).

Twelve QTL were year specific, however the three QTL that were detected in both years exhibited 
consistent impact on trait expression. For the C2 QTL influencing ID, the D allele subpopulations consistently outperformed the $\mathrm{C}$ allele subpopulations (Figure $3 \mathrm{a}$ ). Similarly the B allele subpopulations consistently outperformed the A allele subpopulations at the D2 QTL for SP (Figure 3b). All the QTL detected in both environments were for the traits underlying the commercial trait SY, suggesting that detailed analysis of the determinant traits is a pre-requisite to understanding of the control of more complex traits. Although ID and SP are also, they are less so than SY and were shown to be useful for detection of QTL with consistent effects across environments.

Homozygous allele configurations (AA, BB, CC, and DD) could not be tested at any QTL in this full-sib $\mathrm{F}_{1}$ population structure. For QTL with a consistent impact on performance, such as the C2 QTL for ID, it may be useful to select fixed (DD) genotypes and eliminate the $\mathrm{C}$ allele from elite populations. Subsequent crosses among progeny and testing to determine the influence of homozygous allele configurations will help to determine the type of MAS designs required to optimise commercial seed production.

Among the many allele and QTL combinations observed in the gene mapping population, there were a few specific alleles that account for a substantial proportion of the variation observed. The $\mathrm{B}$ allele at the SP QTL on linkage group D2, and the D allele at the IP QTL on D2 are both examples of single alleles having a massive impact on trait variation, each accounting for up to $50 \%$ of the difference in subpopulation means in both years. Although all the QTL are of fundamental interest in the determination of the genetic control of seed production, these two QTL may have direct commercial applications.

\section{MAS experiment}

Design of DNA tests for the implementation of an MAS proof-of-concept trial must account for the genetic, environmental, and economic factors in a target market. Most cost-effective MAS strategies are used to discard the inferior portion and select the superior portion of a segregating plant population, rather than to pick the absolute best genotype.

This experiment indicated many QTL impact seed production. Examination of QTL magnitude and their allele configuration effects suggested a simple MAS design using tests for only two genetic markers may be adequate to identify superior progeny. The risk of using only the two DNA tests was that allele variation at the 14 unselected QTL might blur the results. However, by using the most influential QTL, we were able to maximise the benefit:cost aspect while minimising that risk.

We used a genetic test for the most influential QTL for
ID and SP. Implementation of this MAS design resulted in a clear stratification of the population, in which the $\mathrm{ID}+\mathrm{SP}+$ subpopulation was statistically superior for SY to the ID-SP- population in both years (Figure 4). Although the subpopulation ID+SP+ exhibited the highest SY trait values in each year, it was statistically identical to a subpopulation with only one positive allele in each environment. In terms of individual plant performance, in SY02 the ID+SP+ subpopulation had 6 of the 10 top ranked plants, and only 1 of the 10 bottom ranked plants among the 92 individuals tested. In SY03, $\mathrm{ID}+\mathrm{SP}+$ had 7 of the top 10 , and none of the bottom 10 plants in the population.

It is envisaged that utilisation of more QTL information could result in better resolution of subpopulation means and individual performance. However, the success rates observed for detecting the most commercially suitable plants using this simple MAS design suggests that it may be the most favourable in terms of benefit:cost ratio. In the commercial use of MAS, these genetic tests would be used in tandem with field trials. As we have shown, the tests used did not select $100 \%$ of the best individuals, but rather selected for the subpopulation with superior performance on average. In a commercial implementation, MAS would be very useful to pre-select for the subpopulation with the highest mean performance, thereby enhancing the opportunity to create varieties with the most robust seed production capability for commercialisation. MAS would also be very useful in the identification of genetically superior parent plants following screening by phenotypic selection.

It is necessary to confirm QTL effects and test MAS designs in new research and commercial white clover populations. These populations must include related and unrelated full-sib structures, and the more complex polycross structures used in commercial breeding programmes. In commercial practice, MAS for seed production must be a cost-effective enhancement of current field trials. At the operational level, simple and reliable methods for DNA sampling must be employed, and breeding designs must be examined to ensure that the best use is made of the power of MAS for the selection of commercially successful plant cultivars. Using genetic tools such as MAS for improvement of commercial traits should result in more efficient breeding programmes and ultimately in cultivars which can capture maximum market share in the forage seed industry.

\section{REFERENCES}

Barrett, B.; Griffiths, A.; Schreiber, M.; Ellison, N.; Mercer, C.; Bouton, J.H.; Ong, B.; Forster, J.; Sawbridge, T.; Spangenburg, G.; Bryan, G.J.; Woodfield, D.R. 2004. A microsatellite map of white clover. Theor. App. Genet. 109: 596-608. 
Caradus, J.R.; Clifford, P.T.P.; Chapman, D.F.; Cousins, G.R.; Williams, W.M.; Miller, J.E. 1997. Breeding and description of Grasslands Sustain. New Zealand Journal of Agricultural Research 40: 1-7.

Faville, M.; Barrett, B.; Griffiths, A.; Schreiber, M.; Mercer, C.; Baird, I.; Ellison, N.; Bryan, G.J.; Woodfield, D.R.; Forster, J.; Ong, B.; Sawbridge, T.; Spangenburg, G.; Easton, H.S. 2003. Implementing molecular marker technology in forage improvement. Proceedings of the New Zealand Grassland Association 65: 229-238.

Mercer, C.F.; Van den Bosch, J.; Miller, K.J. 1999. Effectiveness of recurrent selection of white clover (Trifolium repens) for resistance to New Zealand populations of clover cyst nematode (Heterodera trifolii). Nematology 1: 449-455.

Sawbridge, T.; Ong, E.; Binnion, C.; Emmerling, M.; Meath, K.; Nunan, K.; O’Neill, M.; O’Toole, F.; Simmounds, J.; Wearne, K.; Winkworth, A.;
Spangenburg, G. 2003. Generation and analysis of expressed sequence tags in white clover (Trifolium repens L.). Plant Science 165: 1077-1087.

Tanksley, S.; McCouch, S. 1997. Seed banks and molecular maps: unlocking genetic potential from the wild. Science 277: 1063-1066.

Widdup, K.; Woodfield, D.R.; Baird, I.; Clifford, P. 2004. Response to selection for seed yield in six white clover cultivars. Proceedings of the New Zealand Grassland Association 66: 103-110.

Woodfield, D.R.; Baird, I.; Clifford, P. 2004. Genetic control of white clover seed yield potential. Proceedings of the New Zealand Grassland Association 66: 111-117.

Xiao, J.; Li, J.; Grandillo, S.; Ahn, S.N.; Yuan, L.; Tanksley, S.D.; McCouch, S.R. 1998. Identification of trait-improving quantitative trait loci alleles from a wild rice relative, Oryza rufipogon. Genetics 150: 899909. 